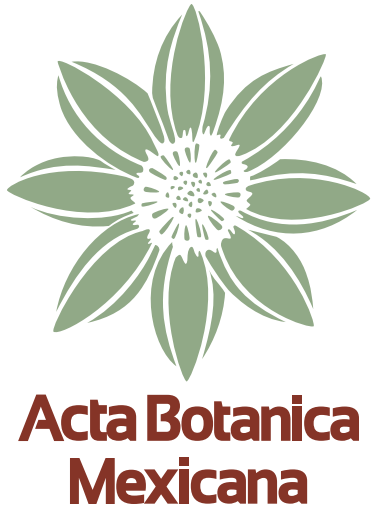

\title{
Fenología de la especie amenazada Leptocereus scopulophilus (Cactaceae) en un bosque semideciduo de Cuba occidental
}

\section{Phenology of the threatened species Leptocereus scopulophilus (Cactaceae) in a semi-deciduous forest of western Cuba}

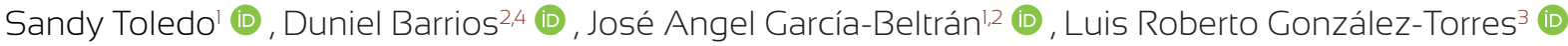

\section{Resumen:}

Antecedentes y Objetivos: Los estudios fenológicos han mostrado su utilidad para entender la dinámica de los ecosistemas y pueden ser útiles para la conservación de especies vegetales. En cactáceas son escasos los estudios que evalúan la fenología reproductiva y vegetativa, en particular sobre especies de bosques semideciduos. El presente estudio aborda diferentes aspectos de la fenología de Leptocereus scopulophilus, una cactácea amenazada y endémica presente en un bosque semideciduo de Cuba occidental. Este estudio tiene como objetivos principales caracterizar la fenología de L. scopulophilus, comparar su comportamiento interanual con diferentes regímenes de precipitación y evaluar las relaciones entre las fenofases y las variables ambientales.

Métodos: Se seleccionaron 50 individuos adultos y se registraron, mensualmente durante tres años, el número de botones, flores, frutos, así como el crecimiento vegetativo y los retrocesos de una rama seleccionada al azar. Se evaluaron mensualmente el porcentaje de iluminación, la temperatura, la humedad relativa y las precipitaciones. Los patrones anuales e interanuales de cada fenofase fueron comparados mediante estadística circular. La relación existente entre variables ambientales y las fenofases se evaluó con una correlación de Spearman.

Resultados clave: Los patrones de floración y fructificación resultaron unimodales con máximos desfasados en un mes. La floración coincidió con el final de la época seca y la fructificación ocurrió al inicio de la época lluviosa. El crecimiento vegetativo fue generalmente de forma aleatoria y simultáneo a la reproducción, mientras el retroceso de las ramas sucedió durante la época lluviosa. La variable más correlacionada con las fenofases fue la iluminación.

Conclusiones: La apertura del dosel del bosque semideciduo donde habita L. scopulophilus favorece la reproducción y el crecimiento de sus ramas. Este evento estuvo modulado por la duración e intensidad de las lluvias de la temporada previa.

Palabras clave: cambios estacionales, crecimiento vegetativo, estadística circular, floración, fructificación, retroceso de las ramas, variables ambientales.

\section{Abstract:}

Background and Aims: Phenological studies have shown their utility in understanding the ecosystem dynamics and could be useful for the conservation of plant species. In cacti, studies evaluating reproductive and vegetative phenology are still scarce, particularly in species inhabiting semi-deciduous forest. The present study addresses different aspects of the phenology of Leptocereus scopulophilus, an endemic threatened cactus inhabiting a semi-deciduous forest of western Cuba. The main objectives of this study are to characterize the phenology of L. scopulophilus, to compare its interannual behavior in years with different rainfall regimes, and to evaluate the relationships between phenophases and environmental variables.

Methods: Fifty adult individuals were selected and the number of buds, flowers, fruits, as well as vegetative growth and retrogressions of a randomly selected branch were recorded monthly for three years. The percentage of lighting, temperature, relative humidity and rainfall was evaluated monthly. The annual and interannual patterns of each phenophase were compared using circular statistics. The relationship between climatic variables and the phenophases was evaluated using a Spearman correlation.

Key results: Flowering and fruiting patterns were unimodal with lagged maximums in one month. Flowering coincided with the late dry season and fruiting occurred at the beginning of the rainy season. Vegetative growth generally occurred randomly and simultaneously with reproduction, while the retrogressions occurred during the rainy season. The variable most correlated with the phenophases was lighting.

Conclusions: The opening of the canopy of the semi-deciduous forest where $L$. scopulophilus occurs favors the reproduction and growth of its branches. This event was modulated by the duration and intensity of the rains of the previous season.

Key words: branch retrogressions, circular statistics, climatic variables, flowering, fruiting seasonal changes, vegetative growth.

${ }^{1}$ Universidad de Concepción, Facultad de Ciencias Naturales y Oceanográficas, Edmundo Larenas 234, Concepción, Chile.

${ }^{2}$ Universidad de La Habana, Jardín Botánico Nacional, carretera "El Rocío" km 31⁄2, Calabazar, Boyeros, 19230 La Habana, Cuba.

${ }^{3}$ University of British Columbia, Department of Botany, Vancouver, Canada.

${ }^{4}$ Autor para la correspondencia: duniel.barrios@ gmail.com
Recibido: 24 de marzo de 2020.

Revisado: 18 de mayo de 2020

Aceptado por Marie-Stéphanie Samain: 28 de agosto de 2020.

Publicado Primero en línea: 8 de septiembre de 2020. Publicado: Acta Botanica Mexicana 128(2021).

c) (i) Este es un artículo de acceso abierto (c) bajo la licencia Creative Commons 4.0 Atribución-No Comercial (CC BY-NC 4.0 Internacional).
Citar como: Toledo, S., D. Barrios, J. A. García-Beltrán y L. R. González-Torres. 2020(2021). Fenología de la especie amenazada Leptocereus scopulophilus (Cactaceae) en un bosque semideciduo de Cuba occidental. Acta Botanica Mexicana 128: e1701. DOI: 10.21829/abm128.2021.1701

e-ISSN: $2448-7589$ 


\section{Introducción}

La fenología es el estudio de los eventos biológicos acomodados a cierto ritmo periódico que ocurren en una especie, en relación con los cambios climáticos de su hábitat (Font-Quer, 1968; Johansson et al., 2015). Es considerada una ciencia ambiental integrativa que ha alcanzado una posición prominente en investigaciones acerca del cambio climático global, debido a la capacidad que ofrece para monitorear, entender y predecir el momento en que ocurren eventos biológicos como la floración, fructificación y crecimiento de las poblaciones de plantas (Rosenzweig et al., 2008). Los estudios fenológicos son importantes para analizar las interacciones ecológicas que se establecen entre las especies y resultan clave para entender la dinámica de los ecosistemas (Johansson et al., 2015).

La variabilidad fenológica de las plantas está determinada por factores ambientales como la precipitación, la humedad, la temperatura y la iluminación, siendo los dos últimos los más estudiados por estar directamente relacionados con la estacionalidad (Elzinga et al., 2007). Adicionalmente, la variabilidad fenológica puede estar influenciada por las respuestas individuales de las especies que conforman la comunidad, debido a diferencias entre individuos conespecíficos, según su estatus fisiológico o localización en determinados microhábitats, e incluso por el comportamiento desigual de las partes de un mismo individuo (WiIliams-Linera y Meave, 2002).

Varios estudios en la última década han abordado la fenología de cactáceas con diferentes fines (Munguía-Rosas y Sosa, 2010; Rojas-Sandoval y Meléndez-Ackerman, 2011; Barrios et al., 2014; Gudiño y De la Barrera, 2014; Giorgis et al., 2015; Matias-Palafox et al., 2017; ArroyoPérez et al., 2019; Salvatierra, 2020). La mayoría de dichas investigaciones poseen como enfoque principal la fenología reproductiva o floral y vinculan otros aspectos como el estudio de sistemas de cruzamiento, éxito reproductivo, sincronía floral, así como la relación del tamaño de las plantas con las fenofases. Solo algunos de estos estudios evalúan la relación existente entre variables ambientales y las fenofases, encontrándose las temperaturas medias y mínimas relacionadas positivamente con la presencia de botones, flores (Ruiz et al., 2000; Munguía-Rosas y Sosa, 2010; Rojas-Sandoval y Meléndez-Ackerman, 2011) y fru- tos (Salvatierra, 2020). Por otro lado, relaciones negativas han sido observadas entre la humedad relativa, con la producción de flores en Stenocereus griseus (Haw.) Buxb. (Ruiz et al., 2000) y frutos de Eulychnia acida Phil. (Salvatierra, 2020), así como entre la temperatura mínima y la producción de botones en Astrophytum ornatum (DC.) Britton \& Rose (Matias-Palafox et al., 2017). Sin embargo, aún son escasos los estudios fenológicos en cactáceas donde se evalúen sistemáticamente el crecimiento (Rosello y Belmonte, 1999) o pérdida de los tallos (fenología vegetativa), así como los factores climáticos desencadenantes de las fenofases de cactáceas que habitan el sotobosque de bosques tropicales semideciduos.

Los bosques semideciduos constituyeron la formación vegetal más extendida en Cuba, que fue desbrozada en su mayor parte para la siembra de caña de azúcar (Funes, 2008). Actualmente se encuentran entre las formaciones vegetales con mayor número de especies amenazadas (González-Torres et al., 2016). Leptocereus scopulophilus Areces es una cactácea endémica de Cuba occidental con tres localidades conocidas; solo una se desarrolla en el sotobosque de un bosque semideciduo mesófilo (Barrios, 2014). Para dicha cactácea, González-Torres et al. (2012) evaluaron el crecimiento vegetativo durante un año y Barrios et al. (2014) la floración durante seis meses. En ambos estudios se considera el aumento de la luz que se produce en el sotobosque por la caída de las hojas durante el período seco, el factor desencadenante de ambas fenofases. Sin embargo, sus consideraciones se basaron en el conocimiento empírico y en el aporte de estudios realizados por Wycherley (1973), Van Schaik (1986) y Lepsch-Cunha y Mori (1999), donde se demuestra que las especies que habitan el sotobosque aumentan la productividad en las épocas con mayor iluminación.

El presente estudio tiene como objetivos principales (1) caracterizar la fenología de Leptocereus scopulophilus, (2) comparar el comportamiento interanual de las fenofases y (3) evaluar la relación de las fenofases (botones, flores, frutos, crecimiento y retroceso de las ramas) con las variables ambientales (precipitación, temperatura, humedad e iluminación) del bosque semideciduo donde se desarrolla la especie. Nosotros hipotetizamos que la iluminación es la variable ambiental más importante en el 
desencadenamiento de la floración y el crecimiento de los tallos de L. scopulophilus.

\section{Materiales y Métodos}

\section{Área de estudio}

La población de Leptocereus scopulophilus se encuentra en el Pan de Matanzas ( $81^{\circ} 41^{\prime} 44.2^{\prime \prime}$ longitud $O$ y $23^{\circ} 01^{\prime} 30^{\prime \prime}$ latitud $\mathrm{N}$ ), cerro mogotiforme, ubicado a $11 \mathrm{~km}$ al oeste de la ciudad de Matanzas, Cuba (CNNG, 2000) (Fig. 1). En la ladera sureste de dicha elevación se desarrolla un bosque semideciduo mesófilo sobre un suelo rocoso de carbonato de calcio (González-Torres et al., 2007). La población de L. scopulophilus se localiza a una altura promedio de 212 m s.n.m. y en un área de $3500 \mathrm{~m}^{2}$ (Barrios et al., 2012a).
El sitio posee un clima tropical estacional con la estación seca en invierno (noviembre-abril) y la lluviosa en verano (mayo-octubre). Las precipitaciones alcanzan $352 \mathrm{~mm}$ en la estación seca y un promedio de temperatura de $23.6{ }^{\circ} \mathrm{C}$. Durante la estación lluviosa las precipitaciones llegan hasta $1070 \mathrm{~mm}$ y un promedio de temperaturas de $25.8^{\circ} \mathrm{C}$ (González-Torres et al., 2012). La humedad relativa oscila entre $82 \%$ en la época de secas y $89.4 \%$ en la época lluviosa (García-Beltrán et al., 2017).

\section{Especie de estudio}

Leptocereus scopulophilus es un cactus arborescente con un patrón de ramas difuso, de hasta $5 \mathrm{~m}$ de altura. Sus ramas principales son erectas, mientras que las más altas
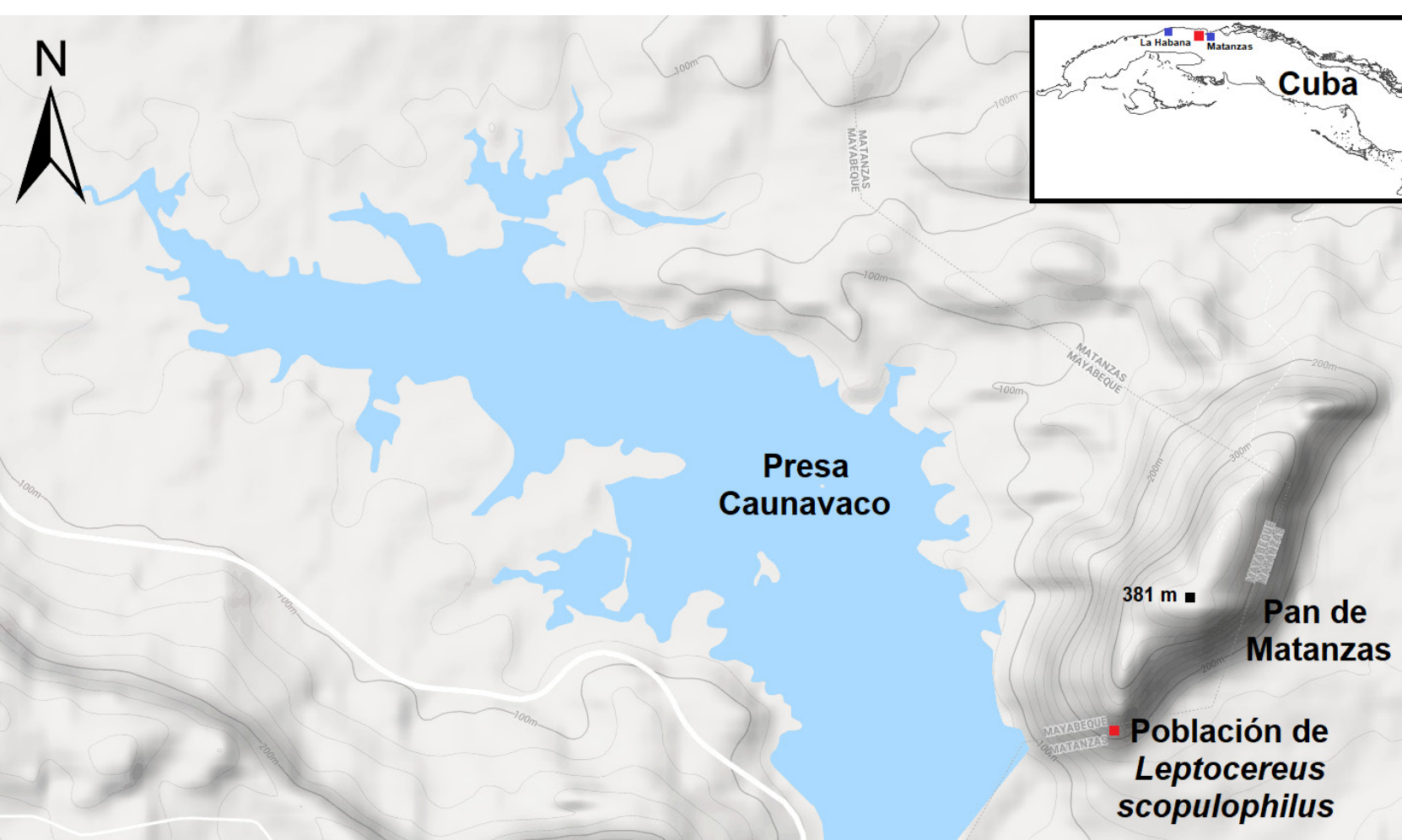

El Palenque :

Figura 1: Localización geográfica del Pan de Matanzas, Cuba. 
son muy ramificadas y tienen un crecimiento plagiótropo oblicuo, con 4-7 costillas aplanadas y crenadas, con espinas agrupadas en las depresiones. Las flores son nocturnas, hermafroditas, con un receptáculo verde, casi tubuloso y espinoso, con el estigma y los estambres exertos (Barrios et al., 2012b). Los individuos de la especie abren 1-3 flores por noche y algunos llegan a un máximo de 19. En la población del Pan de Matanzas existen tres morfotipos florales distinguibles por la coloración de los tépalos: salmón, pardo arenoso y magenta, con diferencias en el número de óvulos (Martínez, 2014). Los frutos son elíptico-globosos, verdes a verde amarillentos con areolas espinosas persistentes en la madurez (Areces, 1993) y pueden contener semillas vivíparas (Barrios et al., 2012a). Leptocereus scopulophilus es una especie xenógama auto-incompatible que necesita de polinizadores para la formación de frutos (Barrios et al., 2012b).

\section{Fenología}

Se seleccionaron al azar 50 individuos adultos previamente enumerados. En cada planta se registró mensualmente el número de botones, flores, frutos nuevos, frutos totales, así como el crecimiento vegetativo y el retroceso de una rama seleccionada al azar, la cual se marcó al inicio del estudio. El crecimiento vegetativo de la rama marcada se consideró como el crecimiento neto y se midió con una cinta métrica ( $\pm 1 \mathrm{~mm}$ ). El retroceso se midió en correspondencia a la longitud de la rama que se secó. Las ramas que se secaron completamente fueron sustituidas por otras, igualmente seleccionadas al azar. Se definió la actividad reproductiva como la frecuencia de individuos florecidos o con producción de frutos nuevos en un mes dado, de acuerdo con Barrios et al. (2014).

\section{Variables ambientales}

El porcentaje de iluminación que llega al sotobosque se evaluó en 16 individuos seleccionados al azar y distribuidos en el área que ocupa la población. En cada uno de ellos se fotografió entre las 09:00 y las 11:00 la cobertura del dosel con una cámara digital Samsung ES95 ubicada 30 $\mathrm{cm}$ al norte de la base de cada individuo. Las fotos fueron procesadas con el programa Adobe Photoshop CS3 (Adobe, 2007), donde el efecto de las nubes se corrigió manual- mente antes de obtener una imagen binaria. El color negro representó la cobertura vegetal y el blanco las zonas sin cobertura vegetal en el dosel (iluminación). La imagen binaria del sistema RGB obtenida se convirtió a porcentaje de iluminación mediante la siguiente formula: \%=Iluminación $(R G B) \times 100 / 255$.

Con un Data Logger (Hobo U23 Pro v. 2, Bourne, EUA) se registraron la temperatura $\left( \pm 0.1^{\circ} \mathrm{C}\right)$ y la humedad relativa $( \pm 1 \%$ ) cada 30 minutos por día. Los datos de septiembre de 2016 a mayo de 2017 se perdieron por afectaciones al sensor producidas por roedores. Las precipitaciones mensuales se obtuvieron del pluviómetro ubicado en la base de la elevación perteneciente a la Empresa de Aprovechamiento Hidráulico "Embalse Caunavaco". Las precipitaciones, la temperatura y la humedad relativa de un mes se consideraron como las ocurridas en el tiempo que transcurrió entre dos muestreos. Se calculó la oscilación térmica diaria como la diferencia que se establece entre la temperatura máxima y mínima en cada uno de los meses, tal como plantea WMO (1992). El estudio se realizó durante tres años consecutivos, desde enero de 2016 y hasta diciembre de 2018.

\section{Análisis de datos}

Se calculó la dirección del vector medio (ángulo medio, $\mu$ ), su longitud $(r)$ y la desviación estándar circular para la producción de botones, flores y frutos, el crecimiento vegetativo y el retroceso de las ramas. La longitud del vector medio varía entre 0 y 1 , valores superiores a 0.5 indican la agrupación de las observaciones alrededor del ángulo medio y la muestra es unimodal. Adicionalmente, la unimodalidad de las observaciones se comprobó mediante pruebas de Rayleigh (Fisher, 1993; Zar, 1999). Para comparar la distribución de las muestras entre los años del estudio, se realizaron pruebas de Mardia-Watson-Wheeler (Fisher, 1993; Zar, 1999). La actividad reproductiva, la producción media de botones, flores y frutos, el crecimiento vegetativo y el retroceso de las ramas entre años se confrontaron mediante una prueba de Watson-Williams F, la cual contrasta los ángulos medios y las longitudes del vector medio (Fisher, 1993; Zar, 1999). Las comparaciones entre años realizadas mediante las dos últimas pruebas se realizaron por pares de años. 
Los eventos fenológicos anteriores, la actividad reproductiva y las variables ambientales se ilustraron por medio de histogramas circulares, donde cada barra corresponde con el número de observaciones mensuales. Cada uno de los días muestreados se convirtió a un ángulo $(\alpha)$ de la escala circular mediante la fórmula $\alpha=\left(360^{\circ}\right)(X) / k$, donde $X$ representa el día del mes en que ocurrió el muestreo y $\mathrm{k}$ el número total de días del año. Todos los análisis anteriores y los histogramas circulares se realizaron en el programa Oriana v. 4.02 (Kovach, 2011), tal como sugiere la metodología propuesta por Morellato et al. (2010) y se consideraron estadísticamente significativos para $\mathrm{p}<0.05$.

Se comprobó la normalidad y homogeneidad de varianza de las variables mediante las pruebas de Kolmogorov Smirnov y Levene, respectivamente. La relación existente entre las variables ambientales y las fenofases se evaluó mediante una correlación de Spearman debido a que no cumplieron las premisas previamente evaluadas. La actividad reproductiva y vegetativa pueden tardar cierto tiempo antes de que se observe una respuesta. Por ello, para la correlación entre la iluminación y las fenofases reproductivas (botones, flores y frutos), los porcentajes de iluminación utilizados correspondieron al del mes previo, mientras que para la correlación entre la iluminación y las fenofases vegetativas (crecimiento y retrocesos), se utilizó la suma de los porcentajes de los dos meses anteriores. Para las corre- laciones con el resto de las variables ambientales (temperatura, humedad y precipitaciones) se utilizaron los datos entre dos muestreos. Las correlaciones se realizaron en el programa Statistica v. 10.0. (StatSoft, 2011) y se consideraron estadística y biológicamente significativas para $p<0.05$ y $r>0.5$, respectivamente, de acuerdo con Morellato et al. (2010).

\section{Resultados}

\section{Patrones fenológicos}

La incorporación de los individuos de Leptocereus scopulophilus a la reproducción comenzó en enero de 2016 y 2017 (Fig. 2), con mayor cantidad de individuos activos en abril (38\%) y marzo (36\%), respectivamente. En 2018 la actividad reproductiva comenzó en febrero y la mayor cantidad de individuos activos se encontró en marzo y abril (28\%) (Fig. 2).

Los botones y las flores presentaron un patrón unimodal (Cuadro 1), aunque pueden ocurrir esporádicamente durante todo el año (Fig. 3). La producción de botones tuvo su media en abril en los tres años del estudio (Cuadro 1), mientras que el máximo de producción se alcanzó en abril durante 2016 y en marzo durante 2017 y 2018 (Figs. 3A-C). La media de producción de flores en 2016 se alcanzó a inicios de mayo (Cuadro 1, Fig. 3D), y difirió significativamente (Cuadro 2) respecto a 2017 y 2018 (Cuadro 1). Durante los
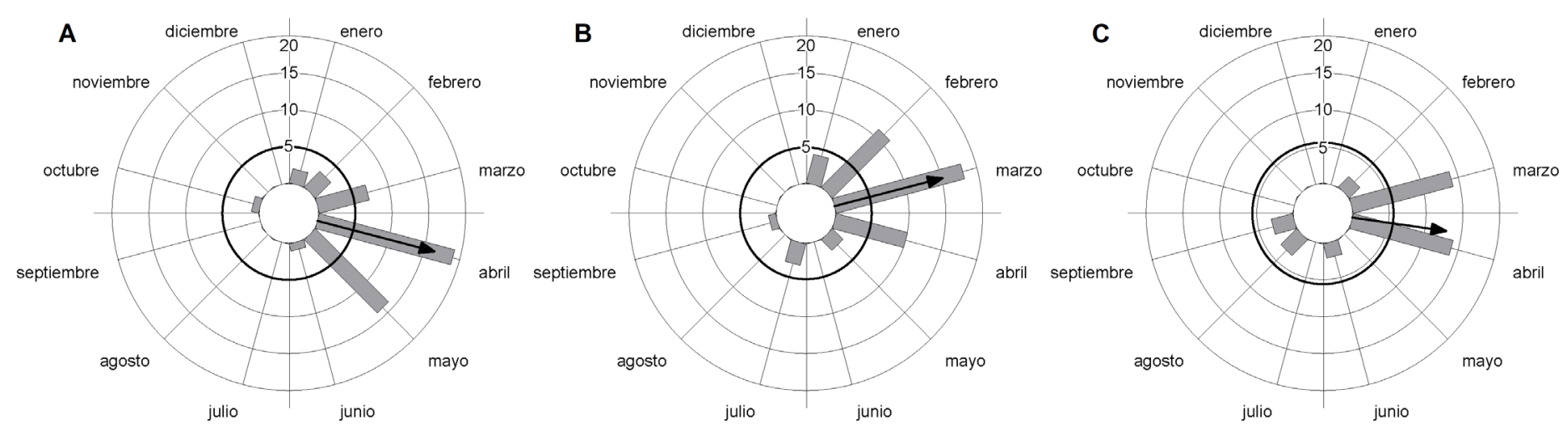

Figura 2: Actividad reproductiva de Leptocereus scopulophilus Areces ( $n=50$ ), en el Pan de Matanzas, Cuba, durante los años 2016 (A), 2017 (B) y 2018 (C). Los círculos de trazos finos delimitan el número de observaciones y las barras se corresponden con el número de individuos observados. La flecha indica el vector medio y el círculo negro el valor crítico de Rayleigh. La ausencia de observaciones en junio de 2017 y mayo de 2018 se debió a la pérdida de datos durante ambos meses. 
Cuadro 1: Producción de botones, flores, frutos nuevos, crecimiento vegetativo y retroceso de las ramas de Leptocereus scopulophilus Areces $(n=50)$, en el Pan de Matanzas, Cuba, 2016-2018.

\begin{tabular}{|c|c|c|c|c|c|c|c|c|c|}
\hline & 2016 & 2017 & 2018 & 2016 & 2017 & 2018 & 2016 & 2017 & 2018 \\
\hline & \multicolumn{3}{|c|}{ Botones } & \multicolumn{3}{|c|}{ Flores } & \multicolumn{3}{|c|}{ Frutos nuevos } \\
\hline $\begin{array}{l}\text { Número de } \\
\text { observaciones }\end{array}$ & 255 & 295 & 160 & 265 & 350 & 68 & 346 & 218 & 175 \\
\hline Ángulo medio $(\mu)$ & $107.25^{\circ}$ & $95.98^{\circ}$ & $95.97^{\circ}$ & $120.05^{\circ}$ & $104.23^{\circ}$ & $107.89^{\circ}$ & $144.48^{\circ}$ & $118.38^{\circ}$ & $158.89^{\circ}$ \\
\hline $\begin{array}{l}\text { Mes correspon- } \\
\text { diente a } \mu\end{array}$ & abril & abril & abril & mayo & abril & abril & mayo & abril & junio \\
\hline $\begin{array}{l}\text { Longitud del vector } \\
\text { medio ( } r)\end{array}$ & 0.88 & 0.86 & 0.71 & 0.90 & 0.89 & 0.58 & 0.67 & 0.64 & 0.47 \\
\hline $\begin{array}{l}\text { Desviación } \\
\text { Estándar Circular }\end{array}$ & $27.96^{\circ}$ & $30.75^{\circ}$ & $46.90^{\circ}$ & $26.06^{\circ}$ & $27.03^{\circ}$ & $59.21^{\circ}$ & $51.20^{\circ}$ & $53.41^{\circ}$ & $69.50^{\circ}$ \\
\hline $\begin{array}{l}\text { Prueba de Rayleigh } \\
\text { (Z) }\end{array}$ & 200.95 & 221.13 & 81.86 & 215.47 & 280.14 & 23.36 & 155.68 & 91.42 & 40.16 \\
\hline \multirow[t]{2}{*}{$\begin{array}{l}\text { Prueba de Rayleigh } \\
\text { (p) }\end{array}$} & $<1 \times 10^{-12}$ & $<1 \times 10^{-12}$ & $<1 \times 10^{-12}$ & $<1 \times 10^{-12}$ & $<1 \times 10^{-12}$ & $7.12 \times 10^{-11}$ & $<1 \times 10^{-12}$ & $<1 \times 10^{-12}$ & $<1 \times 10^{-12}$ \\
\hline & \multicolumn{3}{|c|}{ Crecimiento vegetativo } & \multicolumn{3}{|c|}{ Retroceso de las ramas } & & & \\
\hline Ángulo medio $(\mu)$ & $153.34^{\circ}$ & $139.01^{\circ}$ & $178.43^{\circ}$ & $245.54^{\circ}$ & $322.13^{\circ}$ & $289.08^{\circ}$ & & & \\
\hline $\begin{array}{l}\text { Mes correspon- } \\
\text { diente a } \mu\end{array}$ & junio & mayo & junio & septiembre & noviembre & octubre & & & \\
\hline $\begin{array}{l}\text { Longitud del vector } \\
\text { medio }(r)\end{array}$ & 0.84 & 0.34 & 0.29 & 0.77 & 0.91 & 0.49 & & & \\
\hline $\begin{array}{l}\text { Desviación } \\
\text { Estándar Circular }\end{array}$ & $33.51^{\circ}$ & $84.02^{\circ}$ & $90.18^{\circ}$ & $40.68^{\circ}$ & $24.75^{\circ}$ & $68.34^{\circ}$ & & & \\
\hline $\begin{array}{l}\text { Prueba de Rayleigh } \\
\text { (Z) }\end{array}$ & 389.92 & 19.08 & 22.33 & 64.62 & 363.42 & 113.03 & & & \\
\hline $\begin{array}{l}\text { Prueba de Rayleigh } \\
\text { (p) }\end{array}$ & $<1 \times 10^{-12}$ & $5.13 \times 10^{-9}$ & $2 \times 10^{-10}$ & $<1 \times 10^{-12}$ & $<1 \times 10^{-12}$ & $<1 \times 10^{-12}$ & & & \\
\hline
\end{tabular}

tres años la máxima de producción de flores se alcanzó en abril (Figs. 3D-F). Entre los tres años la distribución de botones y flores se comportó de forma diferente (Cuadro 3).

La producción de frutos nuevos resultó unimodal en 2016 y 2017, mientras que fue multimodal en 2018 (Cuadro 1, Fig. 4C), y ocurrió esporádicamente durante todo el año (Figs. 4A-C). La media de producción de frutos nuevos se alcanzó en meses diferentes en los tres años de estudio (Cuadro 1), mientras que el máximo de producción se obtuvo en mayo de 2016 y en abril durante 2017 y 2018 (Figs. 4A-C). La distribución de la producción de frutos nuevos difirió entre los tres años de estudio (Cuadro 3). Respecto a los frutos totales, en 2016 la mayor disponibilidad ocurrió en mayo, mientras en 2017 y 2018 fue en abril y junio, respectivamente (Figs. 4D-F). La disponibilidad de frutos totales difirió entre 2017 y el resto de los años de estudio (Cuadro 3).

El crecimiento de las ramas durante 2016 resultó unimodal, mientras en el resto de los años fue multimodal (Cuadro 1, Figs. 5A-C). La media de crecimiento en 2016 y 2018 se alcanzó en junio, lo cual no difirió respecto a 2017 (Cuadro 2) pese a que se alcanzó en mayo (Cuadro 1, Figs. 

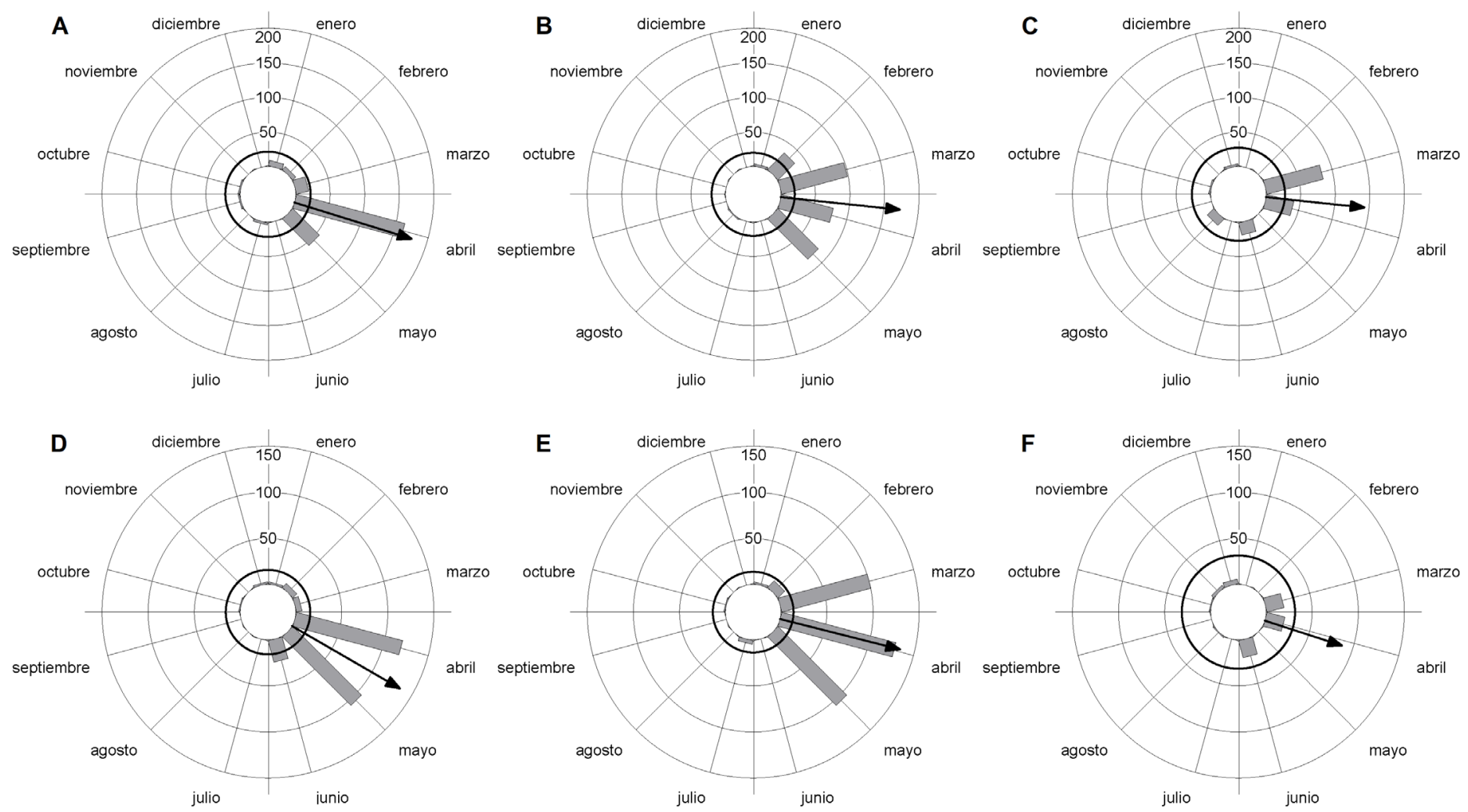

Figura 3: Producción de botones (A-C) y flores (D-F) de Leptocereus scopulophilus Areces ( $n=50)$, en el Pan de Matanzas, Cuba, durante los años 2016 ( $A$ y D), 2017 ( $B$ y E) y 2018 (C y F). Los círculos de trazos finos delimitan el número de observaciones y las barras se corresponden con el total de observaciones mensuales. La flecha indica el vector medio y el círculo negro el valor crítico de Rayleigh. La ausencia de observaciones en junio de 2017 y mayo de 2018 se debió a la pérdida de datos durante ambos meses.

$5 A-C)$. El crecimiento máximo de las ramas (Figs. $5 A-C$ ) y la distribución (Cuadro 3) difirió entre los tres años de estudio.

El retroceso de las ramas fue unimodal durante 2016 y 2017, mientras que en 2018 resultó multimodal (Cuadro 1, Figs. 5D-F). La media de los retrocesos difirió entre los tres años de estudio (Cuadros 1, 2, Figs. 5D-F). El máximo de retroceso en los tres años se alcanzó en diciembre de 2017 (Figs. 5D-F). El retroceso de las ramas se distribuyó de modo diferente entre los tres años de estudio (Cuadro 3).

\section{Variables ambientales}

La máxima iluminación generalmente corresponde con la época de secas, de noviembre a abril (Figs. 6A-C). El porcentaje de iluminación que llega al dosel comenzó su ascenso en noviembre durante 2016 y 2018, sobrepasando 20\%, cifra que se alcanzó ligeramente en diciembre de 2017. Durante los tres años el porcentaje de iluminación aumenta hasta marzo o abril (Figs. 6A-C).
El año con mayor cantidad de precipitaciones fue 2017 (Fig. 6E), con un total de 1715.2 mm; en 2016 y 2018 el total de precipitaciones alcanzado fue de 1203.4 y $1238.9 \mathrm{~mm}$, respectivamente. El momento de mayores precipitaciones coincide con la época de lluvias, que ocurre desde mayo hasta octubre, aunque en 2016 y 2017 se observó un mes con altas precipitaciones en el periodo seco (Figs. 6D, E). Durante 2016 y 2018 el máximo de precipitaciones ocurrió en junio, mientras en 2017 fue en julio (Figs. 6D-F).

La temperatura media osciló entre $23-27{ }^{\circ} \mathrm{C}$ y de manera general los mayores valores de temperaturas ocurrieron en 2018 , entre 29 y $39{ }^{\circ} \mathrm{C}$ (Figs. 6G-I). Los picos de temperatura máxima se concentraron principalmente durante el invierno en 2018 (Fig. 6I), que corresponde con la época seca, aunque en 2016 las máximas fueron superiores durante la época lluviosa y en 2017 no se observó un patrón claro por la ausencia de datos en la mayor parte de 
Cuadro 2: Comparación por pares de años mediante la prueba de Watson-Williams F de la dirección del ángulo medio de la producción de botones, flores y frutos nuevos, así como el crecimiento vegetativo y el retroceso de las ramas de Leptocereus scopulophilus Areces ( $\mathrm{n}=50$ ), en el Pan de Matanzas, Cuba, 2016-2018.

\begin{tabular}{lccc}
\hline & Años a comparar (cantidad de observaciones) & F & p \\
\hline \multirow{3}{*}{ Botones } & $2016(255)-2017(295)$ & 0.05 & 0.82 \\
& $2016(255)-2018(160)$ & 2.51 & 0.11 \\
\hline \multirow{3}{*}{ Flores } & $2017(295)-2018(160)$ & 1.90 & 0.16 \\
& $2016(265)-2017(350)$ & 6.65 & 0.01 \\
\hline \multirow{3}{*}{ Frutos nuevos } & $2016(265)-2018(68)$ & 5.19 & 0.02 \\
& $2017(350)-2018(68)$ & 1.49 & 0.22 \\
\hline & $2016(346)-2017(218)$ & 15.16 & $1.11 \times 10^{-4}$ \\
Crecimiento vegetativo & $2016(346)-2018(175)$ & 1.90 & 0.16 \\
& $2017(218)-2018(175)$ & 15.97 & $7.66 \times 10^{-5}$ \\
\hline & $2016-2017$ & 1.59 & 0.20 \\
Retroceso de las ramas & $2016-2018$ & 0.21 & 0.64 \\
& $2017-2018$ & 1.03 & 0.30 \\
\hline
\end{tabular}
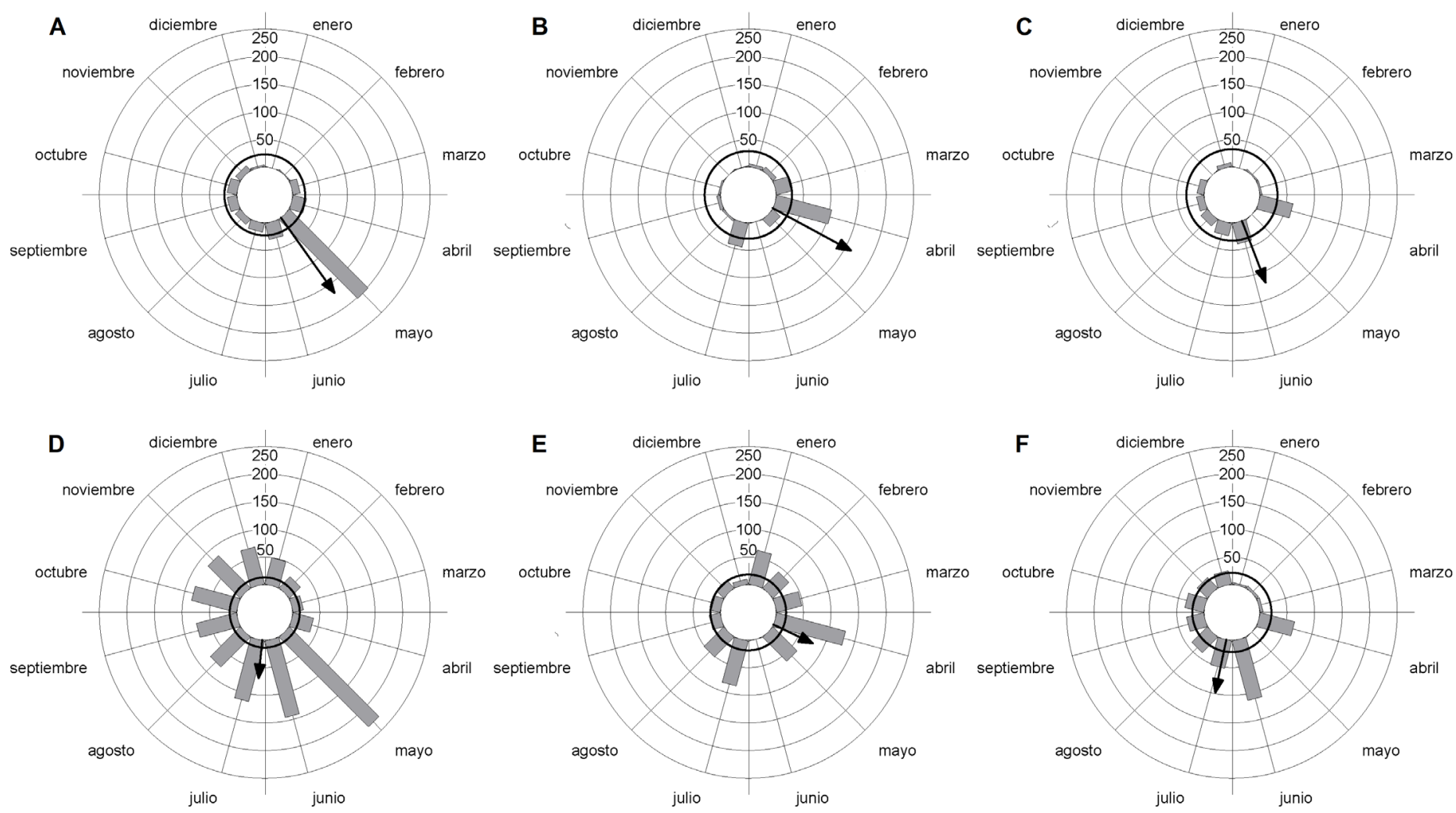

Figura 4: Producción de frutos nuevos (A-C) y frutos totales (D-F) de Leptocereus scopulophilus Areces ( $n=50$ ), en el Pan de Matanzas, Cuba, durante los años 2016 (A y D), 2017 (B y E) y 2018 (C y F). Los círculos de trazos finos delimitan el número de observaciones y las barras se corresponden con el total de observaciones mensuales. La flecha indica el vector medio y el círculo negro el valor crítico de Rayleigh. La ausencia de observaciones en junio de 2017 y mayo de 2018 se debió a la pérdida de datos durante ambos meses. 
Cuadro 3: Comparación por pares de años mediante la prueba de Mardia-Watson-Wheeler de la distribución de botones, flores, frutos nuevos, frutos totales, crecimiento vegetativo y retroceso de las ramas de Leptocereus scopulophilus Areces ( $n=50)$, en el Pan de Matanzas, Cuba.

\begin{tabular}{lccc}
\hline & Años comparados (cantidad de observaciones) & W & p \\
\hline \multirow{3}{*}{ Botones } & $2016(255)-2017(295)$ & 110.95 & $<1 \times 10^{-12}$ \\
& $2016(255)-2018(160)$ & 80.24 & $<1 \times 10^{-12}$ \\
& $2017(295)-2018(160)$ & 78.32 & $<1 \times 10^{-12}$ \\
\hline \multirow{3}{*}{ Flores } & $2016(265)-2017(350)$ & 14.25 & $8.04 \times 10^{-4}$ \\
& $2016(265)-2018(68)$ & 15.03 & $5.43 \times 10^{-4}$ \\
& $2017(350)-2018(68)$ & 51.86 & $5.47 \times 10^{-12}$ \\
\hline \multirow{3}{*}{ Frutos nuevos } & $2016(346)-2017(218)$ & 122.40 & $<1 \times 10^{-12}$ \\
& $2016(346)-2018(175)$ & 56.99 & $<1 \times 10^{-12}$ \\
Frutos totales & $2017(218)-2018(175)$ & 26.83 & $1.49 \times 10^{-6}$ \\
\hline Crecimiento & $2016(1005)-2017(535)$ & 59.70 & $<1 \times 10^{-12}$ \\
vegetativo & $2016(1005)-2018(395)$ & 2.60 & 0.272 \\
& $2017(535)-2018(395)$ & 47.84 & $4.08 \times 10^{-11}$ \\
\hline Retroceso & $2016(255)-2017(295)$ & 137.89 & $<1 \times 10^{-12}$ \\
de las ramas & $2016(255)-2018(160)$ & 45.03 & $1.66 \times 10^{-10}$ \\
\hline & $2017(295)-2018(160)$ & 47.70 & $4.38 \times 10^{-11}$ \\
\hline
\end{tabular}

los meses. Por otra parte, la humedad relativa media osciló entre 75 y $90 \%$, mientras que las mayores variaciones se encontraron en la humedad relativa mínima, la cual varió entre 25 y $75 \%$ (datos no mostrados).

Relación entre las fenofases y las variables ambientales

La variable más correlacionada con las fenofases de Leptocereus scopulophilus fue la iluminación. Otras variables de temperatura y humedad relativa estuvieron correlacionadas solamente con una o dos fenofases en 2016 y 2018, que fueron los años donde se obtuvieron la mayoría de las correlaciones, debido a que en 2017 los datos perdidos correspondieron al periodo reproductivo. Las precipitaciones solo se correlacionaron con la producción de frutos en 2018 (Cuadro 4).

La iluminación se correlacionó positivamente con la producción de botones, flores, frutos y crecimiento de las ramas, y negativamente con el retroceso de las ramas du- rante 2016 y 2018 (Cuadro 4). Tanto la temperatura mínima como la media, de 2016, se correlacionaron positivamente con el retroceso de las ramas (Cuadro 4). La temperatura media en 2018 lo estuvo negativamente con el crecimiento vegetativo. La temperatura máxima se correlacionó solo con la producción de flores de 2018, mientras la oscilación térmica diaria en 2016 lo hizo positivamente con la producción de botones (Cuadro 4). La humedad relativa mínima y media mostró correlación negativa con la producción de botones y flores en 2018 (Cuadro 4).

\section{Discusión}

\section{Actividad reproductiva}

La actividad reproductiva de Leptocereus scopulophilus comprende mayormente la época seca, lo que concuerda con lo reportado por Barrios et al. (2014) para la especie. En contraste con dichos autores, nosotros registramos una actividad reproductiva de los individuos inferior a $40 \%$ (Fig. 2) en la época de mayor actividad (febrero-mayo), mientras 

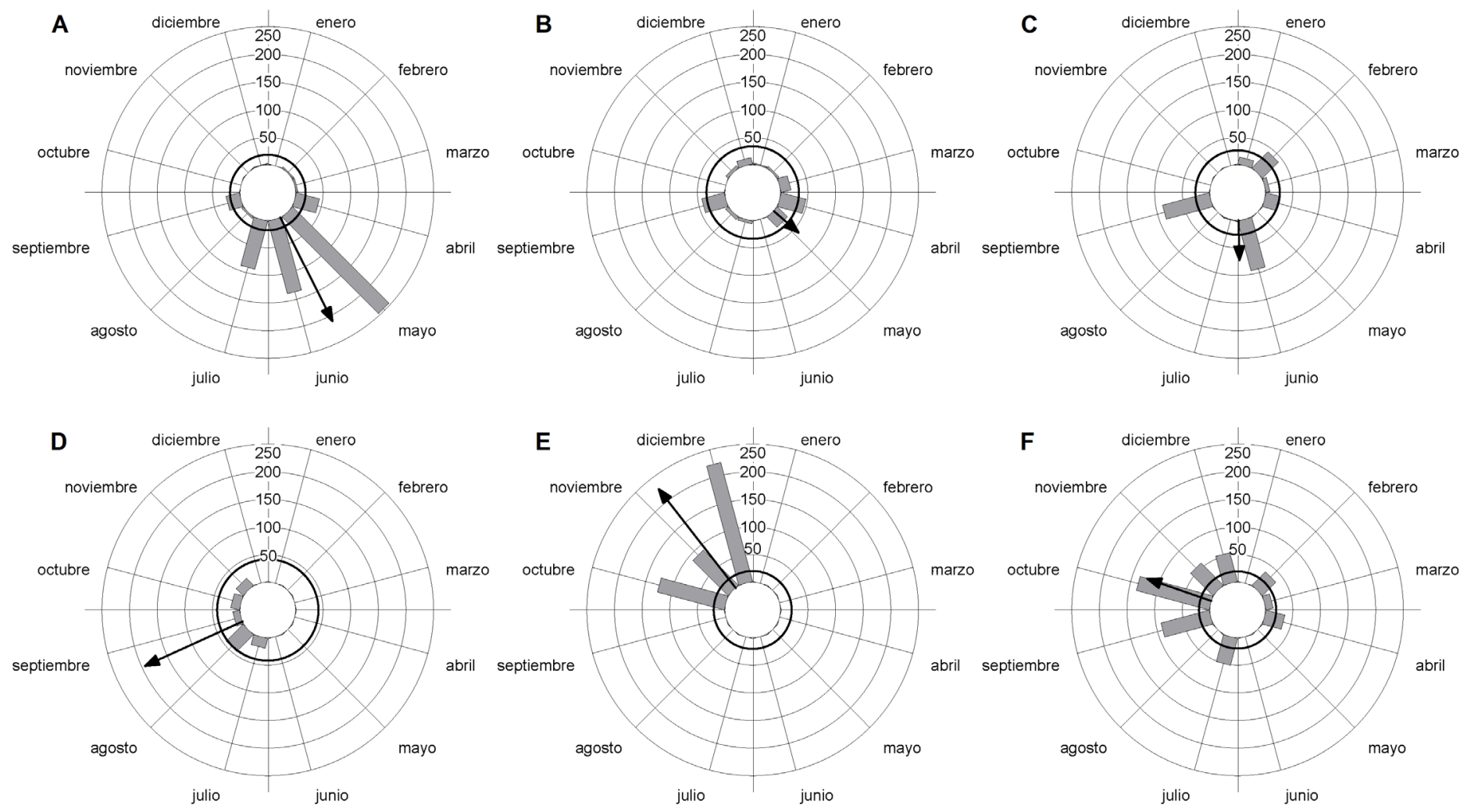

Figura 5: Crecimiento vegetativo (A-C) y retroceso de las ramas (D-F) de Leptocereus scopulophilus Areces ( $n=50)$, en el Pan de Matanzas, Cuba, durante los años 2016 ( y D), 2017 (B y E) y 2018 (C y F). Los círculos de trazos finos delimitan el crecimiento y el retroceso en cm. Las barras se corresponden con el crecimiento o retroceso en $\mathrm{cm}$. La flecha indica el vector medio y el círculo negro el valor crítico de Rayleigh. La ausencia de observaciones en junio de 2017 y mayo de 2018 se debió a la pérdida de datos durante ambos meses.

que Barrios et al. (2014) reportan en la misma época valores entre $60-80 \%$.

Varias causas podrían explicar las diferencias entre nuestros resultados y los de Barrios et al. (2014). Resulta poco probable que desde 2009 hayan sucedido cambios climáticos drásticos en el área de estudio, pues en 2008 (año previo) precipitaron $1556.4 \mathrm{~mm}$ y en 2009, 1351.4 $\mathrm{mm}$ (D. Barrios, datos no publicados), cantidades similares a las observadas durante 2016-2018. Probablemente la baja actividad reproductiva registrada esté relacionada con el envejecimiento poblacional, o las constantes pudriciones de las ramas que predominaron respecto al crecimiento durante 2017 y 2018. Otra explicación posible pudiera estar dada por el aumento o la constancia en el tiempo de los niveles de herbivoría reportados para la especie por Barrios (2008), Barrios et al. (2012c) y Martínez (2014), causando un efecto negativo sobre la producción de estructuras reproductoras.

\section{Floración}

La floración unimodal (Fig. 3) en Leptocereus scopulophilus durante el final de la época de secas y el inicio de la lluviosa (Figs. 6D-F) concuerda con lo referido para la especie por Areces (2003) y Barrios et al. (2014), así como con el patrón general de floración de la mayoría de las cactáceas cubanas (Barrios et al., 2015). Patrones de floración unimodal durante la época seca también han sido registrados en cactáceas de bosques secos como Pachycereus pringlei Britton \& Rose (Fleming et al., 2001), Stenocereus queretaroensis (F.A.C. Weber ex Mathes.) Buxb. (Ibarra-Cerdeña et al., 2005), Pterocereus gaumeri Britton \& Rose (Méndez et al., 2005) y Eulychnia acida (Salvatierra, 2020).

El retraso de la producción de botones y flores en 2016 respecto a 2017-2018 (Fig. 3) podría ser el resultado del comportamiento desigual de las condiciones climáticas durante los tres años analizados. Al inicio de 2016 (enero-febrero) ocurrió una ampliación de la época lluvio- 

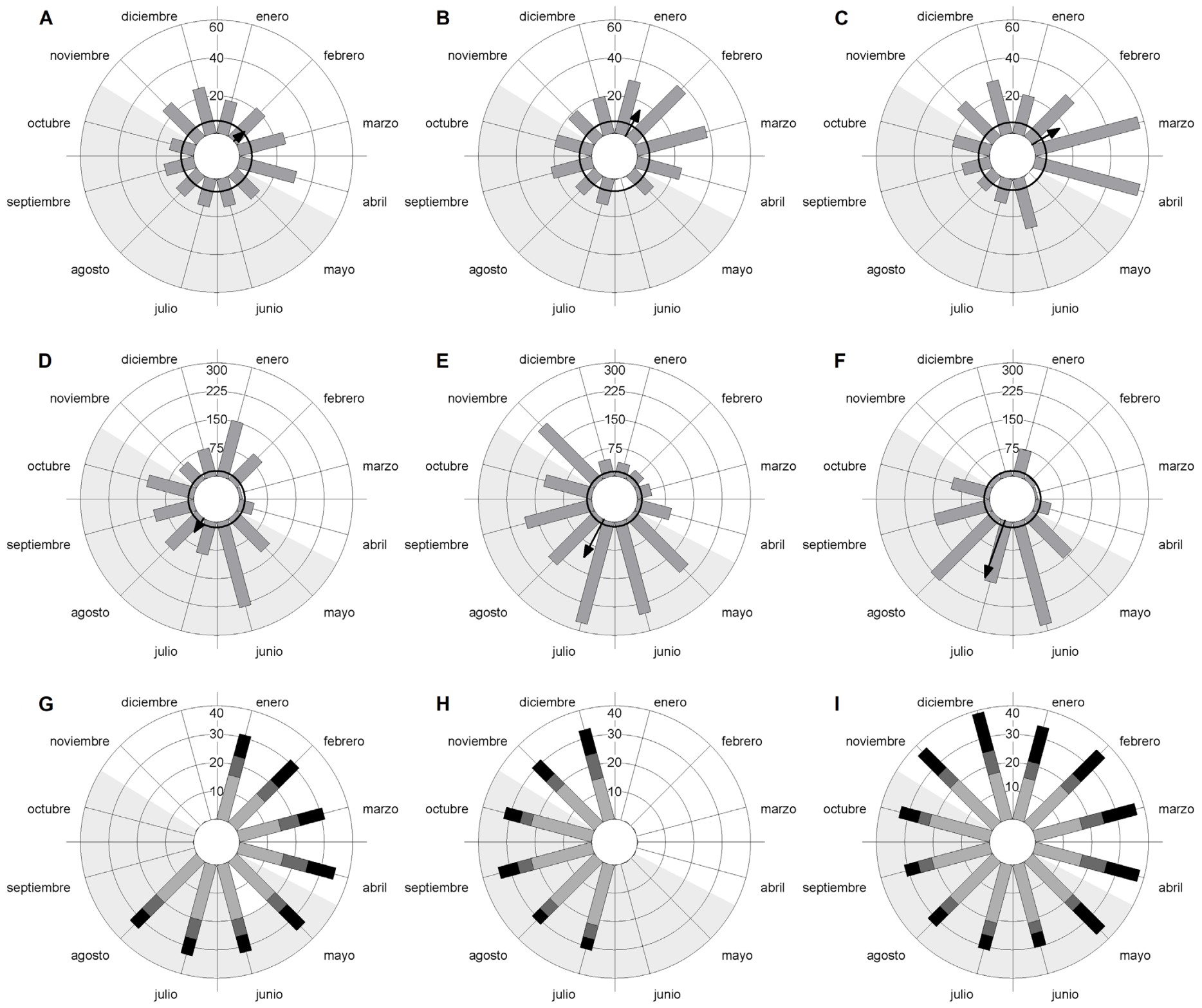

Figura 6: Variables climáticas en el área que ocupa la población de Leptocereus scopulophilus Areces en el Pan de Matanzas, Cuba, durante los años $2016(A, D, G), 2017$ (B, E, H) y 2018 (C, F, I). Las barras se corresponden con la iluminación en \% (A-C), precipitaciones en mm (D-F) y temperatura en ${ }^{\circ} \mathrm{C}$ (G-I). La zona sombreada representa la época lluviosa de acuerdo con Lecha et al. (1994). Las diferentes intensidades de gris en las barras de las figuras G-I representan la temperatura media (gris oscuro), máxima (negro) y mínima (gris claro) mensual. La ausencia de barras en junio de 2017 (B), mayo de 2018 (C), de septiembre a diciembre de 2016 (G) y de enero a mayo de 2017 (H) se debió a la pérdida de datos durante dichos meses.

sa, que se unió a las precipitaciones de los últimos meses de 2015 (datos no mostrados) (Fig. 6D), lo cual no ocurrió durante los primeros meses de 2017 y 2018 (Figs. 6E, F). Según Cutié et al. (2015), desde marzo de 2015 comenzaron a desarrollarse las condiciones oceánicas propias del evento El Niño-Oscilación del Sur (ENOS), lo que alcanzó su máximo desarrollo en diciembre del propio año y fue uno de los tres más intensos de las últimas seis décadas. La principal influencia del evento ENOS sobre Cuba ocurre en el período seco, donde el clima se torna lluvioso y en ocasiones tormentoso, como ocurrió en 2015 y 2016 (Cutié et al., 2015; 2016). Por ello, 2016 presenta las mayores precipitaciones en invierno respecto a 2017 y 2018 (Figs. 6D-F). 
Cuadro 4: Matriz de correlación entre fenofases de Leptocereus scopulophilus Areces $(n=50)$ y variables climáticas en el Pan de Matanzas, Cuba, 2016-2018. Se muestran los valores de coeficiente de correlación de Spearman $\left(r_{s}\right)$ para cada par de variables y se destacan en negritas aquellas estadísticas $(p<0.05)$ y biológicamente $\left(r_{s}>0.5\right)$ significativas. La ausencia de datos se debe a su pérdida durante el año.

\begin{tabular}{|c|c|c|c|c|c|c|c|c|c|c|c|c|c|c|c|}
\hline \multirow[t]{2}{*}{ Variables climáticas } & \multicolumn{3}{|c|}{$\begin{array}{l}\text { Producción } \\
\text { de botones }\end{array}$} & \multicolumn{3}{|c|}{$\begin{array}{l}\text { Producción } \\
\text { de flores }\end{array}$} & \multicolumn{3}{|c|}{$\begin{array}{c}\text { Producción } \\
\text { de frutos nuevos }\end{array}$} & \multicolumn{3}{|c|}{$\begin{array}{r}\text { Crecimiento } \\
\text { vegetativo }\end{array}$} & \multicolumn{3}{|c|}{$\begin{array}{c}\text { Retroceso } \\
\text { de las ramas }\end{array}$} \\
\hline & 2016 & 2017 & 2018 & 2016 & 2017 & 2018 & 2016 & 2017 & 2018 & 2016 & 2017 & 2018 & 2016 & 2017 & 2018 \\
\hline Iluminación & 0.64 & 0.80 & 0.53 & 0.88 & 0.86 & 0.61 & 0.31 & 0.69 & 0.01 & 0.67 & 0.66 & 0.33 & -0.60 & -0.20 & -0.61 \\
\hline Precipitaciones & -0.22 & -0.35 & -0.02 & 0.00 & -0.34 & -0.25 & 0.17 & -0.28 & 0.58 & 0.06 & 0.27 & 0.08 & 0.00 & 0.17 & -0.17 \\
\hline $\begin{array}{l}\text { Temperatura } \\
\text { mínima }\end{array}$ & -0.56 & & -0.27 & -0.45 & & -0.27 & 0.30 & & 0.09 & 0.49 & & -0.29 & 0.73 & & 0.48 \\
\hline $\begin{array}{l}\text { Temperatura } \\
\text { media }\end{array}$ & -0.52 & & 0.01 & -0.33 & & 0.04 & 0.38 & & 0.24 & 0.52 & & -0.58 & 0.76 & & 0.51 \\
\hline $\begin{array}{l}\text { Temperatura } \\
\text { máxima }\end{array}$ & 0.28 & & 0.49 & 0.33 & & 0.59 & 0.61 & & -0.25 & 0.59 & & -0.03 & 0.26 & & 0.11 \\
\hline $\begin{array}{l}\text { Oscilación térmica } \\
\text { diaria }\end{array}$ & 0.79 & & 0.45 & 0.50 & & 0.44 & -0.14 & & -0.25 & -0.32 & & 0.02 & -0.48 & & -0.10 \\
\hline $\begin{array}{l}\text { Humedad relativa } \\
\text { mínima }\end{array}$ & -0.37 & & -0.60 & -0.13 & & -0.65 & -0.04 & & 0.20 & 0.01 & & -0.04 & -0.21 & & 0.18 \\
\hline $\begin{array}{l}\text { Humedad relativa } \\
\text { media }\end{array}$ & -0.22 & & -0.44 & -0.30 & & -0.53 & -0.28 & & 0.30 & 0.20 & & 0.13 & -0.21 & & -0.08 \\
\hline
\end{tabular}

Según Barrios et al. (2014), durante la época seca en el Pan de Matanzas la mayoría de los árboles pierden sus hojas y muchas de las lianas mueren, lo que posibilita la llegada de mayor cantidad de luz y aumento de las temperaturas en el sotobosque. Ambos aumentos en iluminación y temperatura máxima (que dependen de la intensidad y duración de las lluvias previas) parecen actuar como disparadores de la floración en Leptocereus scopulophilus. Principalmente la iluminación se correlacionó positivamente con la producción de botones y flores (Cuadro 4), y concuerda con lo sugerido por González-Torres et al. (2012) y Barrios et al. (2014). Nuestros resultados muestran un posible umbral de iluminación alrededor de $20 \%$ para que se inicie la floración. Por ejemplo, en 2016, se alcanzó $20 \%$ en febrero (Fig. 6 A), por lo que pudo ser el causante del retraso de la floración respecto a 2017 y 2018 (Fig. 3).

Tal dependencia entre el nivel de luz que atraviesa el dosel y el comienzo de la floración concuerda con el criterio de Adams et al. (1999), quienes consideran que la floración se incrementa linealmente con el aumento de la iluminación y la temperatura. De igual modo, Wycherley (1973), Van Schaik (1986) y Lepsch-Cunha y Mori (1999) demostraron que la productividad aumenta cuando hay mayor luminosidad en especies tropicales del sotobosque. Taiz y Zeiger (2002) consideran que esta relación se debe a la acumulación de recursos durante los días con mayor iluminación, los cuales son utilizados para la floración. Por ello, Leptocereus scopulophilus aprovecha el aumento de la iluminación y florece en la época seca (Fig. 3).

El año con menor floración fue 2018, pese a presentar precipitaciones similares a 2016 y niveles de iluminación similares en 2017. Esta situación podría estar relacionada con que previo a la floración de 2018, los retrocesos ocurridos en 2017 fueron los mayores durante los tres años de estudio (Fig. 5E), donde el balance entre crecimiento y retroceso fue negativo. Tales retrocesos, que fueron máximos en diciembre de 2017 (Fig. 5E), probablemente empeoraron el estado fitosanitario de los individuos, afectando así en 2018 la actividad reproductiva. 


\section{Fructificación}

La concentración de la floración hacia los meses previos a la época lluviosa permite la posterior maduración de los frutos, dispersión de las semillas y el establecimiento de las plántulas en la época de lluvias (Bustamante y Búrquez, 2005). Dicho fenómeno podría aumentar las probabilidades de supervivencia en un momento del año donde el estrés hídrico es mínimo, tal como sugieren Barrios et al. (2014) y García-Beltrán (2014). En regiones tropicales con clima estacional, la disponibilidad de agua en el suelo constituye el principal factor que limita la germinación de las semillas y el establecimiento de las plántulas (Franco, 2002; Baskin y Baskin, 2014). Por ello, en tales ecosistemas la germinación al inicio de la temporada lluviosa es crucial para el reclutamiento de las plantas, permitiendo que las semillas desarrollen un sistema radicular eficiente o almacenen suficientes recursos para sobrevivir a la siguiente temporada seca (Franco, 2002; Oliveira, 2008).

En los dos primeros años del estudio ocurre una caída abrupta de los frutos entre los meses de mayo-junio y abril-mayo, respectivamente. Ello pudiera responder al aborto de los frutos inmaduros producto de las lluvias. Según Vílchez et al. (2008), las precipitaciones pueden dañar o desprender las estructuras reproductoras durante tormentas de lluvias y vientos asociados. El aborto de botones asociado a eventos de lluvias fuertes ha sido observado en Stenocereus griseus (Petit, 2001) y Harrisia portoricensis Britton (Rojas-Sandoval y Meléndez-Ackerman, 2011), y concuerda con la tendencia observada que presentaron la mayoría de las fenofases de correlacionarse negativamente con las precipitaciones (Cuadro 4).

\section{Crecimiento vegetativo y retroceso de las ramas} El crecimiento vegetativo (Figs. $5 \mathrm{~A}-\mathrm{C}$ ), simultáneo a la floración (Fig. 3) y la fructificación (Fig. 4) en Leptocereus scopulophilus, contrasta con una de las características más extendidas en las cactáceas columnares. En este grupo de cactus, el crecimiento vegetativo ocurre durante la época seca, pero no durante la reproducción para alcanzar un mayor ajuste a las limitaciones de agua (Pimienta-Barrios y Nobel, 1995, 1998; Pimienta-Barrios et. al., 2004). El crecimiento vegetativo en $L$. scopulophilus ocurre cuando la temperatura promedio y las precipitaciones descienden durante la época de secas, al igual que Pimienta-Barrios et al. (2004) encontraron para Stenocereus queretaroensis. De acuerdo con Oliveira (2008), el crecimiento durante la época de reproducción puede ser ventajoso debido a que potencia la producción de recursos fotosintéticos durante los primeros estadios del proceso reproductivo. Por tanto, el crecimiento vegetativo simultáneo a la reproducción podría constituir una adaptación de L. scopulophilus para aprovechar la alta iluminación, tal como lo demuestran las correlaciones entre crecimiento e iluminación (Cuadro 4).

El alto número de retrocesos de los tallos registrados (Figs. 5D-F) podría estar influenciado por las precipitaciones, debido a que ocurren principalmente durante o posterior a la época de lluvias. Aunque los retrocesos se contabilizan cuando la rama se seca, su efecto ocurre un tiempo antes, pues el proceso de pudrición tarda aproximadamente un mes. Ello concuerda con la correlación positiva existente entre el retroceso de las ramas y las temperaturas mínima y media durante 2016 (Cuadro 4) y la correlación negativa existente entre la iluminación y el retroceso de las ramas en 2016 y 2018 (Cuadro 4). Esta situación se debe a que los retrocesos ocurren durante la época de lluvias, correspondientes con el verano, donde las temperaturas mínima y media tienden a ascender, y la iluminación es mínima.

\section{Conclusiones}

Leptocereus scopulophilus presenta un patrón reproductivo unimodal, con variaciones interanuales, influenciado por las variables ambientales que inciden en el área. La iluminación es la variable más correlacionada con sus fenofases reproductivas y se encuentra modulada por la duración e intensidad de las precipitaciones de los meses previos. El crecimiento vegetativo ocurre generalmente de forma aleatoria. Se inicia durante la época seca y de forma simultánea a la reproducción, lo cual podría constituir una adaptación de la especie para aprovechar la alta iluminación y compensar los retrocesos que ocurren durante la época de lluvias. Adicionalmente, el retroceso de las ramas es unimodal y ocurre principalmente a finales de la época lluviosa y se extiende hasta inicios del periodo seco, afectando de forma negativa la actividad reproductiva de L. scopulophilus. 


\section{Contribución de autores}

ST y DB concibieron y diseñaron el estudio. ST, DB, JAGB y LRGT contribuyeron con la adquisición de datos, su análisis e interpretación. ST escribió la primera versión del manuscrito. Todos los autores contribuyeron a la discusión, revisión y aprobación del manuscrito final.

\section{Financiamiento}

El presente estudio contó con el apoyo de Planta!-Plantlife Conservation Society y el Jardín Botánico Nacional de la Universidad de La Habana, mediante los proyectos Conservation of endemic relict plants in dry ecosystems y Taxonomía de la familia Cactaceae: una contribución a su conservación.

\section{Agradecimientos}

Agradecemos el apoyo del Jardín Botánico Nacional de la Universidad de La Habana, el de la Sociedad Cubana de Botánica, y Planta! Se agradece la valiosa ayuda de Jorge L. Guerra durante la toma de datos en el campo y la de los trabajadores del Embalse Caunavaco por el apoyo recibido durante las expediciones, en especial a Onel Alfonso por proveer la información de las precipitaciones. Finalmente agradecemos a dos revisores anónimos y a la editora Marie-Stéphanie Samain por sus valiosos comentarios que mejoraron la versión inicial de este manuscrito.

\section{Literatura citada}

Adams, S. R., S. Pearson, P. Hadley y W. M. Patefield. 1999. The effects of temperature and light integral on the phases of photoperiod sensitivity in Petunia $\times$ hybrida. Annals of Botany 83(3): 263-269. DOI: https://doi.org/10.1006/ anbo.1998.0817

Adobe. 2007. Adobe Photoshop CS3, version 10. Adobe Systems Incorporated. San José, EUA. www.adobe.com/products/ photoshop.html

Areces, A. 1993. Leptocereus scopulophilus (Cactaceae), a new species from western Cuba. Brittonia 45(3): 226-230.

Areces, A. 2003. Leptocereus (A. Berger) Britton and Rose: a monographic study of a West Indian genus of Cactaceae (Cactoideae) Inferences from Morphological and Molecular Data. Tesis de doctorado. University of New York. Nueva York, USA. 533 pp.
Arroyo-Pérez, E., C. L. Jiménez-Sierra, J. A. Zavala-Hurtado, J. Flores-Rivas e I. H. Salgado-Ugarte. 2019. Fenología, sincronía floral y éxito reproductivo de Neolloydia conoidea (Cactaceae). Botanical Sciences 97(4): 579-587. DOI: https://doi.org/10.17129/botsci.2184

Barrios, D. 2008. Biología de la polinización de Leptocereus scopulophilus (Cactaceae) en el Pan de Matanzas, Cuba. Tesis de diploma. Facultad de Biología, Universidad de La Habana. La Habana, Cuba. 50 pp.

Barrios, D. 2014. Leptocereus scopulophilus. Bissea 8 (número especial 1): 89-90.

Barrios, D., L. R. González-Torres y J. A. García-Beltrán. 2012a. Vivipary in Cuban cacti: a pioneer study in Leptocereus scopulophilus. Bradleya 30: 147-150. DOI: https://doi. org/10.25223/brad.n30.2012.a17

Barrios, D., L. R. González-Torres y A. Palmarola. 2012b. Biología de la polinización de Leptocereus scopulophilus (Cactaceae) en el Pan de Matanzas, Cuba. Revista del Jardín Botánico Nacional 32-33: 163-168.

Barrios, D., C. A. Mancina y L. R. González-Torres. 2012c. Evidencias de dispersión de semillas de plantas nativas por Capromis pilorides (Rodentia: Capromydae). Revista del Jardín Botánico Nacional 32-33: 315-317.

Barrios, D., L. R. González-Torres y A. Palmarola. 2014. Dinámica de la floración de Leptocereus scopulophilus (Cactaceae): estrategias de atracción. Revista del Jardín Botánico Nacional 34-35: 61-66.

Barrios, D., L. R. González-Torres y A. Palmarola. 2015. Desafíos en el estudio y conservación de las cactáceas cubanas. Bissea 9 (número especial 1): 2.

Baskin, C. C. y J. M. Baskin. 2014. Seeds: ecology, biogeography, and evolution of dormancy and germination. 2nd ed. Academic Press. San Diego, USA. 1602 pp.

Bustamante, E. y A. Búrquez. 2005. Fenología y biología reproductiva de las cactáceas columnares. Cactáceas y Suculentas Mexicanas 50(3): 68-88.

CNNG. 2000. Diccionario Geográfico de Cuba. Oficina Nacional de Hidrografía y Geodesia. La Habana, Cuba. 386 pp.

Cutié, V., M. Hernández, D. Boudet, I. González, V. Guevara, A. León, C. González, C. Fonseca, B. Lapinel, A. Alonso, R. Pérez y J. Bolufé. 2015. Enero-diciembre. Boletín de la Vigilancia del Clima 27(1-12). 
Cutié, V., M. Hernández, D. Boudet, I. González, V. Guevara, A. León, C. González, C. Fonseca, B. Lapinel, A. Alonso, R. Pérez y J. Bolufé. 2016. Enero-diciembre. Boletín de la Vigilancia del Clima 28: 1-12.

Elzinga, J. A., A. Atlan, A. Biere, L. Gigord, A. E. Weis y G. Bernasconi. 2007. Time after time: flowering phenology and biotic interactions. Trends in Ecology and Evolution 22(8): 432-439. DOI: https://doi.org/10.1016/j.tree.2007.05.006

Fisher, N. I. 1993. Statistical analysis of circular data. Cambridge University Press. Cambridge, UK. 269 pp.

Fleming, T. H., C. T. Sahley, J. N. Holland, J. D. Nason y J. L. Hamrick. 2001. Sonoran Desert columnar cacti and the evolution of generalized pollination systems. Ecological Monographs 71(4): 511-530. DOI: https://doi.org/10.1890/00129615(2001)071[0511:SDCCAT]2.0.CO;2

Font-Quer, P. 1968. Diccionario de Botánica. Editorial Labor. Barcelona, España. 1244 pp.

Franco, A. C. 2002. Ecophysiology of woody plants. In: Oliveira, P. S. y R. J. Marquis (eds.). The cerrados of Brazil: ecology and natural history of a neotropical savanna. Columbia University Press. New York, USA. 178-197.

Funes, R. 2008. From rainforest to cane field in Cuba: an environment history since 1492. The University of North Carolina Press. North Carolina, USA. 358 pp.

García-Beltrán, J. A. 2014. Heterogeneidad seminal y viviparidad en Leptocereus scopulophilus (Cactaceae) en el Pan de Matanzas, Cuba. Tesis de diploma. Facultad de Biología, Universidad de La Habana. La Habana, Cuba. 56 pp.

García-Beltrán, J. A., D. Barrios y A. Cuza-Pérez. 2017. Heteromorphism in seeds of Leptocereus scopulophilus (Cactaceae) from Pan de Matanzas, Cuba. Seed Science Research 27: 311-320. DOI: https://doi.org/10.1017/ S0960258517000289

Giorgis, M. A., A. M. Cingolani y D. E. Gurvich. 2015. Flowering phenology, fruit set and seed mass and number of five coexisting Gymnocalycium (Cactaceae) species from Córdoba mountain, Argentina. Journal of the Torrey Botanical Society 142(3): 220-230. DOI: https://doi. org/10.3159/TORREY-D-14-00017.1

González-Torres, L. R., A. Palmarola, Y. Echemendía y D. Barrios. 2007. Conservation of Leptocereus scopulophilus and Leptocereus wrightii, two endemic cacti from Cuba. CactusWorld 25(supplement): 15-20.
González-Torres, L. R., D. Barrios y A. Palmarola. 2012. The ecology and natural history of Leptocereus scopulophilus (Cactaceae). CactusWorld 30(2): 110-114.

González-Torres, L. R., A. Palmarola, L. González-Oliva, E. R. Bécquer, E. Testé, M. A. Castañeira-Colomé, D. Barrios, J. L. Gómez-Hechavarría, J. A. García-Beltrán, L. Granado, D. Rodríguez-Cala, R. Berazaín y L. Regalado (Comp.). 2016. Lista Roja de la flora de Cuba. Bissea 10(número especial 1): 33-283.

Gudiño, W. y E. De la Barrera. 2014. Fenología reproductiva y tolerancia a altas temperaturas en Stenocereus queretaroensis. Polibotánica 37: 63-78.

Ibarra-Cerdeña, C. N., L. Iñiguez-Dávalos y V. Sánchez-Cordero. 2005. Pollination ecology of Stenocereus queretaroensis (Cactaceae), a chiropterophilus columnar cactus, in a tropical dry forest of Mexico. American Journal of Botany 92(3): 503-509. DOI: https://doi.org/10.3732/ajb.92.3.503

Johansson, J., J. Nilsson y N. Jonzén. 2015. Phenological change and ecological interactions: an introduction. Oikos 124: 1-3. DOI: https://doi.org/10.1111/oik.02077

Kovach, W. L. 2011. Oriana-Circular Statistics for Windows, ver. 4. Kovach Computing Services. Pentraeth, UK. http://www. kovcomp.com (consultado enero de 2020).

Lepsch-Cunha, N. y S. A. Mori. 1999. Reproductive phenology and mating potential in a low-density tree population of Couratari multiflora (Lecythidaceae) in central Amazonia. Journal of Tropical Ecology 15(1): 97-121. DOI: https://doi. org/10.1017/S0266467499000693

Martínez, D. 2014. Morfos florales en Leptocereus scopulophilus (Cactaceae) y su éxito reproductivo en el Pan de Matanzas, Cuba. Tesis de diploma. Facultad de Biología, Universidad de La Habana. La Habana, Cuba. 44 pp.

Matias-Palafox, M. L., C. L. Jiménez-Sierra, J. Golubov y M. C. Mandujano. 2017. Reproductive ecology of the threatened star cactus Astrophytum ornatum (Cactaceae): a strategy of continuous reproduction with low success. Botanical Sciences 95(2): 245-258. DOI: https://doi.org/10.17129/ botsci. 780

Méndez, M., R. Durán, A. Dorantes, G. Dzib, L. Simá, P. Simá y R. Orellana. 2005. Floral demography and reproductive system of Pterocereus gaumeri, a rare columnar cactus endemic to Mexico. Journal of Arid Environments 62(3): 363-376. DOI: https://doi.org/10.1016/j.jaridenv.2004.12.002 
Morellato, L. P. C., L. F. Alberti y I. L. Hudson. 2010. Applications of circular statistics in plant phenology: a case studies approach. In: Hudson, I. L. y M. R. Keatley (eds.). Phenological Research: methods for environmental and climate change analysis. Springer. London, UK. Pp. 339-359.

Munguía-Rosas, M. A. y V. J. Sosa. 2010. Phenology of Pilosocereus leucocephalus (Cactaceae, tribe Cereeae): a columnar cactus with asynchronous pulsed flowering. Plant Ecology 211: 191-201. DOI: https://doi.org/10.1007/s11258-0109784-z

Oliveira, P. E. 2008. Fenologia e biologia reprodutiva das espécies do Cerrado. In: Sano, S. M., S. P. Almeida y J. F. Ribeiro (eds.). Cerrado: Ecologia e Flora, Vol. 1. Empresa Brasileira de Pesquisa Agropecuária (EMBRAPA). Brasilia, D.F., Brasil. Pp. 274-290.

Petit, S. 2001. The reproductive phenology of three sympatric species of columnar cacti on Curaçao. Journal of Arid Environments 49(3): 521-531. DOI: https://doi.org/10.1006/ jare.2001.0801

Pimienta-Barrios, E. y P. S. Nobel. 1995. Reproductive characteristics of pitayo (Stenocereus queretaroensis) and their relationship with soluble sugars and irrigation. Journal of the American Society for Horticultural Science 120(6): 1082-1086. DOI: https://doi.org/10.21273/ JASHS.120.6.1082

Pimienta-Barrios, E. y P. S. Nobel. 1998. Vegetative, reproductive, and physiological adaptations to aridity of pitayo Stenocereus queretaroensis (Cactaceae). Economic Botany 52: 401-411.

Pimienta-Barrios, E., S. Mena y C. Robles. 2004. Relaciones entre carbohidratos de reserva, azúcares reductores, crecimiento y fenología del Pitayo de Querétaro (Stenocereus queretaroensis (Weber) Buxbaum). Revista Fitotecnia Mexicana 27(1): 101-106.

Rojas-Sandoval, J. y E. Meléndez-Ackerman. 2011. Reproductive phenology of the Caribbean cactus Harrisia portoricensis: rainfall and temperature associations. Botany 89(12): 861871. DOI: https://doi.org/10.1139/B11-072

Rosello, N. E. y S. E. Belmonte. 1999. Fenología de Browningia candelaris (Meyen) Britton \& Rose en la Quebrada de Cardones, Norte de Chile. Idesia 17: 47-55.
Rosenzweig, C., D. Karoly, M. Vicarelli, P. Neofotis, Q. Wu, G. Casassa, A. Menzel, T. L. Root, N. Estrella, B. Seguin, P. Tryjanowski, C. Liu, S. Rawlins y A. Imeson. 2008. Attributing physical and biological impacts to anthropogenic climate change. Nature 453(7193): 353-357. DOI: https://doi. org/10.1038/nature06937

Ruiz, A., M. Santos, J. Cavelier y P. J. Soriano. 2000. Estudio fenológico de Cactáceas en el Enclave Seco de la Tatacoa, Colombia. Biotropica 32(3): 397-407. DOI: https://doi. org/10.1111/j.1744-7429.2000.tb00486.x

Salvatierra, A. 2020. Reproductive phenology of the arborescent cactus Eulychnia acida Phil. Under three agroecological conditions in the Coquimbo Region, Chile. Chilean Journal of Agricultural Research 80(2): 253-262. DOI: https://doi. org/10.4067/S0718-58392020000200253

StatSoft, Inc. 2011. STATISTICA (data analysis software system), version 10. http://www.statsoft.com (consultado enero de 2020).

Taiz, L. y E. Zeiger. 2002. Plant Physiology. 3a ed. Sinauer Associates. Sunderland, USA. 623 pp.

Van Schaik, C. P. 1986. Phenological changes in a Sumatran rain forest. Journal of Tropical Ecology 2(4): 327- 347. DOI: https://doi.org/10.1017/S0266467400000973

Vílchez, B., R. L. Chazdon y W. Alvarado. 2008. Fenología reproductiva de las especies del dosel en bosques secundarios y primarios de la región Huetar Norte de Costa Rica y su influencia en la regeneración vegetal. Revista Forestal Mesoamericana Kurú 5(15): 16-33.

Williams-Linera, G. y J. Meave. 2002. Patrones fenológicos. In: Guariguata, R. M. y G. H. Kattan (eds.). Ecología y conservación de bosques neotropicales. Libro Universitario Regional. San José, Costa Rica. Pp. 591-624.

WMO. 1992. International meteorological vocabulary No. 182. World Meteorological Organization. Ginebra, Suiza. 784 pp.

Wycherley, P. 1973. The phenology of plants in the sumid tropics. Micronesica 9(1): 75-96.

Zar, J. H. 1999. Biostatistical analysis. Prentice Hall. Princeton, USA. 663 pp. 